\title{
Reverse knuckle guidewire insertion technique for endoscopic ultrasound-guided hepaticogastrostomy using a novel 0.025 -inch guidewire
}

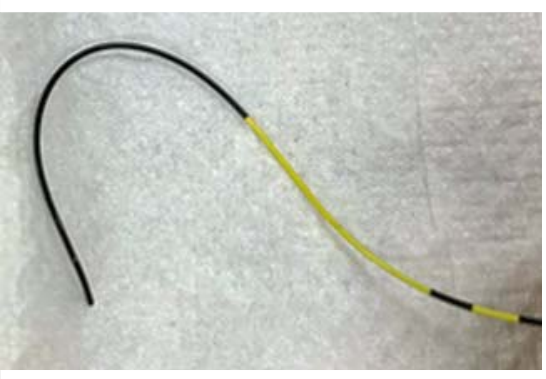

> Fig. 1 Photograph of the novel 0.025inch guidewire (MICHISUJI; KANEKA Medical) that is available in Japan.
Endoscopic ultrasound-guided hepaticogastrostomy (EUS-HGS) is one of the biliary drainage options for patients in whom endoscopic retrograde cholangiopancreatography (ERCP) fails [1,2]. Technical tips for EUS-HGS are divided into several steps [3]. Among these steps, guidewire manipulation is one of most challenging parts of EUS-HGS [4]. In achieving successful guidewire insertion, selection of the guidewire is an important issue. A novel, 0.025 -inch guide- wire has recently become available in Japan (MICHISUJI; KANEKA Medical, Osaka, Japan) (\Fig.1). High flexibility, which is one of the characteristics of this guidewire, plays an especially important role in the safe guidewire technique, which we call "knuckle guidewire insertion" [5]. Herein, we describe technical tips for "reverse" knuckle guidewire insertion during EUS-HGS.

A 78-year-old man underwent percutaneous transhepatic biliary drainage be-
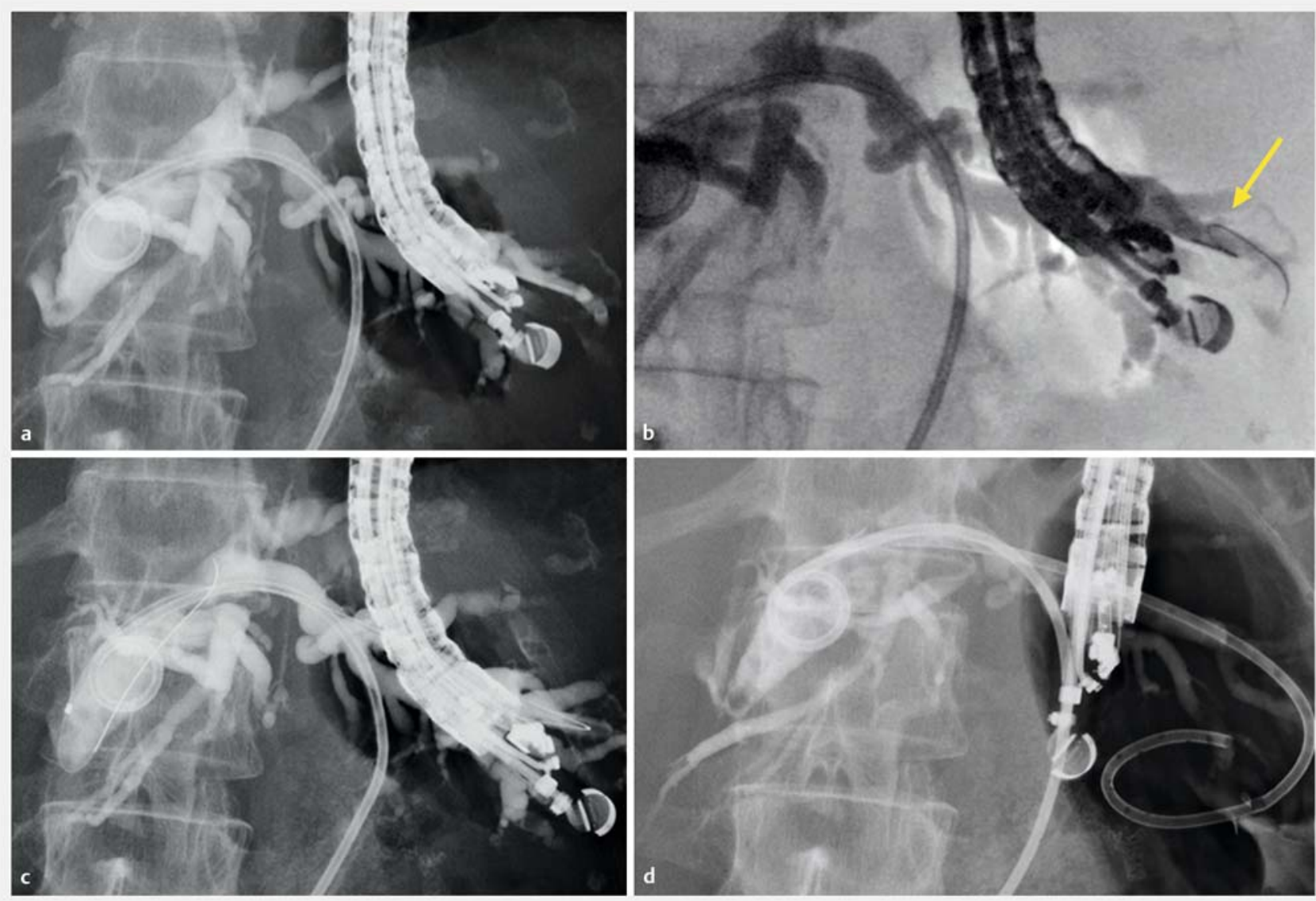

Fig. 2 Fluoroscopic images showing: a contrast medium being injected after the intrahepatic bile duct had been punctured using a $19-G$ needle; $\mathbf{b}$ the reverse knuckle shape successfully made with the guidewire; $\mathbf{c}$ the endoscopic retrograde cholangiopancreatography catheter inserted to dilate the fistula; $\mathbf{d}$ the endoscopic ultrasound-guided hepaticogastrostomy proceeding with placement of a plastic stent. 


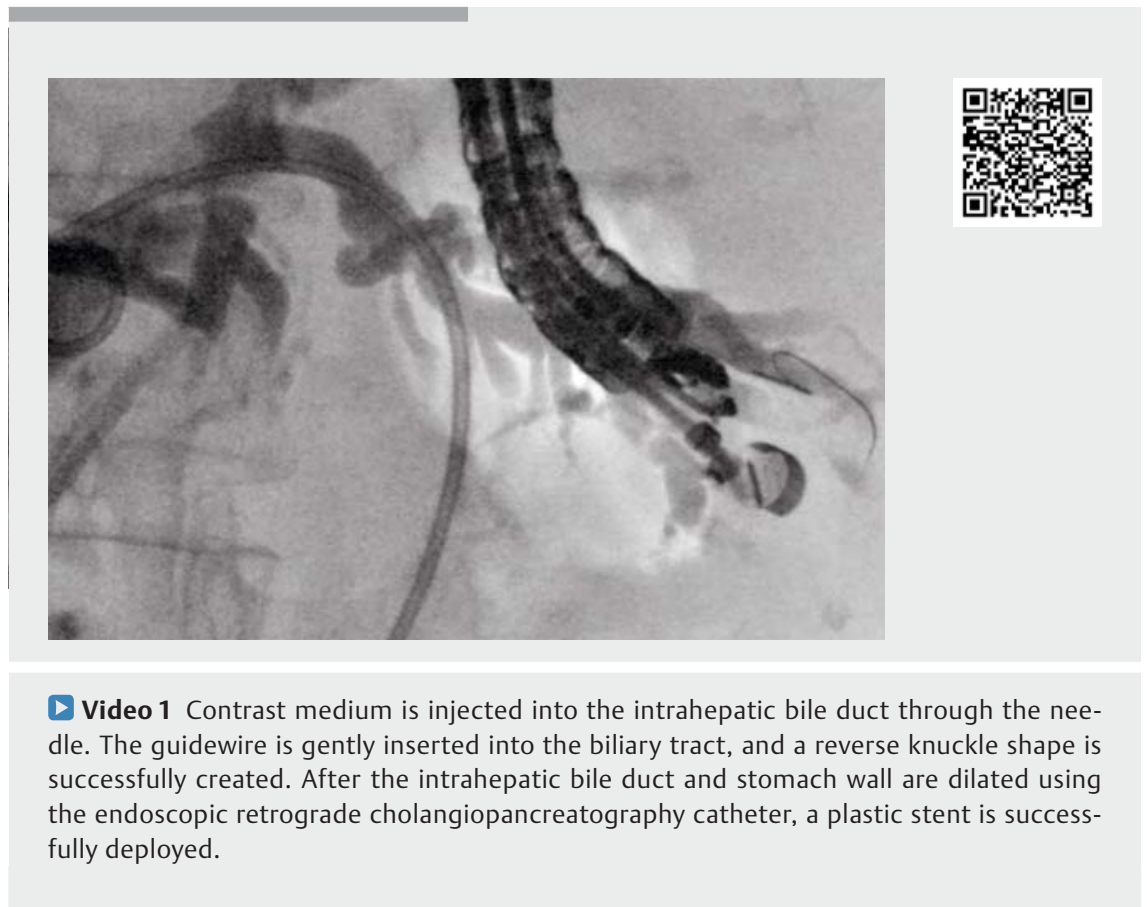

cause of a hepaticojejunostomy stricture. However, internal drainage failed, and he was referred to our hospital for EUS-HGS. The intrahepatic bile duct was identified under EUS guidance but, because the left hepatic parenchyma was enlarged, the direction of EUS scope advancement was from the opposite side of the hepatic hilum. The intrahepatic bile duct was punctured using a $19 \mathrm{G}$ needle, and contrast medium was injected ( $\triangleright$ Fig. 2 a; - Video 1). The novel MICHISUJI guidewire was selected for advancement of the guidewire into the hepatic hilum. As shown in Fig. $\mathbf{2}$ b,a reverse knuckle shape was successfully made because of the flexibility of this novel guidewire, and the guidewire was inserted into the hepatic hilum. After the bile duct and stomach wall had been dilated using the ERCP catheter ( $\triangleright$ Fig. $\mathbf{2}$ c), plastic stent deployment was successfully performed without any adverse events ( $\mathbf{F i g} \mathbf{2} \mathbf{2 d}$ ).

As in the present case, if the direction of EUS scope advancement is from the opposite side of the hepatic hilum, reverse knuckle guidewire insertion using this

novel guidewire may be useful for advancement of the guidewire into the hepatic hilum.

Endoscopy_UCTN_Code_TTT_1AS_2AD

Competing interests

The authors declare that they have no conflict of interest.

The authors

Takeshi Ogura, Masanori Yamada, Tadahiro Yamada, Saori Ueno, Kazuhide Higuchi 2nd Department of Internal Medicine, Osaka Medical College, Osaka, Japan

\section{Corresponding author}

\section{Takeshi Ogura, MD}

2nd Department of Internal Medicine, Osaka Medical College , 2-7 Daigakuchou,

Takatsukishi, Osaka 569-8686, Japan Fax: +81-726846532
[1] Guo J, Giovannini M, Sahai AV et al. A multiinstitution consensus on how to perform EUS-guided biliary drainage for malignant biliary obstruction. Endosc Ultrasound 2018; 7: 356-365

[2] Boulay BR, Lo SK. Endoscopic ultrasoundguided biliary drainage. Gastrointest Endosc Clin N Am 2018; 28: 171-185

[3] Ogura T, Higuchi K. Technical tips for endoscopic ultrasound-guided hepaticogastrostomy. World J Gastroenterol 2016; 21: 3945-3951

[4] Vila J], Pérez-Miranda M, Vazquez-Sequeiros E et al. Initial experience with EUS-guided cholangiopancreatography for biliary and pancreatic duct drainage: a Spanish national survey. Gastrointest Endosc 2012; 76: 1133-1141

[5] Ogura T, Yamada M, Nishioka N et al. Knuckle guidewire insertion: Safe techniques of guidewire insertion into the pancreatobiliary tract using a novel 0.025 -inch guidewire (with video). J Gastroenterol Hepatol 2019. doi:10.1111/jgh. 14895

oguratakeshi0411@yahoo.co.jp

\section{Bibliography}

Endoscopy 2020; 52: E418-E419

DOI 10.1055/a-1149-8738

published online 24.4.2020

(c) 2020. Thieme. All rights reserved.

Georg Thieme Verlag KG, Rüdigerstraße 14, 70469 Stuttgart, Germany

\section{ENDOSCOPY E-VIDEOS}

https://eref.thieme.de/e-videos

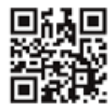

Endoscopy E-Videos is a free access online section, reporting on interesting cases and new

techniques in gastroenterological endoscopy. All papers include a high quality video and all contributions are freely accessible online.

This section has its own submission website at https://mc.manuscriptcentral.com/e-videos
ISSN 0013-726X 